\title{
$\lambda$ RNA Internal Standards Quantify Sensitivity and Amplification Efficiency of Mammalian Gene Expression Profiling
}

BioTechniques 25:504-514 (September 1998)

\author{
Roger D. Madison ${ }^{1,2}$ and \\ Grant A. Robinson ${ }^{1}$ \\ ${ }^{1}$ Duke University Medical \\ Center and ${ }^{2}$ Research Service of \\ the Veterans Affairs Medical \\ Center, Durham, NC, USA
}

\section{INTRODUCTION}

Tremendous progress is being made in compiling DNA sequences for several different genomes, including that of the human (e.g., the Human Genome Project). Because the genes in all somatic cells of an individual are identical, it is the differential expression of the proteins encoded by particular genes that determines a cell's phenotype. There is now increasing interest in relating the simultaneous expression patterns of multiple proteins to complex biological processes. The goal is to develop "expression profiles" of the proteins that are active within a cell during a particular condition. Of the emerging technologies for expression profiling $(3,8,9)$, many require large amounts of starting material [e.g., micrograms of poly(A) RNA] and as such are not useful to laboratories such as ours in which the interest is in single rat nerves and identified cell populations from retina. Such limited tissue requires a technology that is able to amplify the endogenous mRNA population. There are only a few approaches to accomplish such amplification. The two most widely used methods are the polymerase chain reaction (PCR), resulting in exponential amplification, and the linear amplification procedure known as amplified antisense RNA (aRNA), developed by Eberwine and colleagues $(1,3,5,6,8)$. Both have advantages and disadvantages. The most important disadvantages for using the PCR to look at expression levels of multiple mRNAs include a disproportionate decrease in the amplification of larger cDNAs in relation to smaller cDNAs and differences in the efficiency of amplification of mRNAs of the same length. Although similar types of disadvantages must be considered with amplified antisense technology (see below), any differences with this amplification technique will be linear rather than exponential as with the PCR. In addition, most studies of mRNA levels using PCR analyze a single mRNA at a time because of the extreme complexity of reaction conditions needed to carry out multiplex PCR.

Any technology using the aRNA procedure must acknowledge three potential problems: (i) what is the typical amplification and sensitivity of detection for the technique? That is, how much aRNA can be synthesized from limited amounts of template, and how many copies of a given mRNA must be present in the starting material to be reliably detected on the final reverse Northern blot? (ii) How similar is the magnitude of amplification for mRNAs of different length? (iii) How severe is the inherent 3 ' bias for the amplified products due to the oligo(dT) priming of reverse transcription (RT)?

Previous work $(5,8)$ using the aRNA procedure has provided indirect estimates of the sensitivity and potential influence of the problems listed above. This report is the first to study these issues in a direct manner using poly(A) RNA internal standards synthesized from lambda phage $(\lambda)$ DNA. Com- 
bined with optimized conditions yielding millionfold amplification, the poly(A) RNA internal standards could be detected at the level of 10 copies. These optimized conditions and internal standards should prove useful to the many laboratories interested in studying gene expression in limited samples of mammalian tissue. Preliminary results of these experiments have been reported in abstract form (7).

\section{MATERIALS AND METHODS}

\section{cDNA Templates for $\lambda$ RNA Internal Standards}

Three nonoverlapping fragments $(0.4,1.2$ and $2.5 \mathrm{kbp}$ ) of $\lambda$ DNA (Promega, Madison, WI, USA) were isolated and subcloned.

入0.4-pBS. $\lambda$ DNA was digested with PstI. After $1 \%$ SeaPlaque ${ }^{\circledR}$ agarose electrophoresis (FMC BioProducts, Rockland, ME, USA), the appropriate band ( $\lambda$ 4370-4709) was excised, and the fragment was recovered using AgarACE ${ }^{\circledR}$ Enzyme (Promega) digestion and Microcon ${ }^{\circledR}-100$ spin-column purification (Millipore, Bedford, MA, USA). To add 30 3' T:A residues, the fragment was ligated into the PstI site of pSP64A (Promega). After HindIII/ EcoRI digestion of $\lambda 0.4-p S P 64 A$, the new T:A residue-containing fragment was subcloned into pBluescript $^{\circledR}$ II SK(-) (Stratagene, La Jolla, CA, USA) using the same restriction sites and oriented so that in vitro transcription using the T7 promoter yielded transcripts with a $3^{\prime}$ poly(A) tail.

ג1.2-pBS. $\lambda$ DNA was digested with $\mathrm{SacI}$. After agarose electrophoresis, the appropriate band ( $\lambda 24772$ 25877 ) was excised, the fragment recovered as above and ligated into the SacI site of pSP64A. After HindIII/ EcoRI digestion of $\lambda 1.2$-pSP64A, the new fragment was subcloned into the same restriction sites of pBluescript II.

$\lambda$ 2.5-pBS. $\lambda$ DNA was digested with PstI. After agarose electrophoresis, the appropriate band ( $\lambda$ 1183514294 ) was excised, the fragment recovered as above and ligated into the PstI site of pSP64A. After HindIII/ EcoRI digestion of $\lambda 2.5$-pSP64A, the new fragment was subcloned into the same restriction sites of pBluescript II.

$5^{\prime} \lambda$ 2.5-pBS. A $5^{\prime}$ fragment of $\lambda$ DNA from $\lambda 2.5$-pBS was isolated and subcloned to address the issue of $3^{\prime}$ bias. $\lambda 2.5-\mathrm{pBS}$ was digested with HindIII/StuI to excise the $5^{\prime}$-most 600bp fragment of $\lambda$ DNA ( $\lambda$ 1183512 434). After agarose electrophoresis, the appropriate band was excised and subcloned into the HindIII site of pBluescript II.

\section{Preparation and Blotting of Reverse Northern Blots}

The above phagemid vectors containing $\lambda$ DNA were used to transform competent bacterial host strains, and blue/white color screening was used to select several positive colonies that were grown in small liquid cultures. Phagemid DNA from these minipreps was subjected to restriction analysis to verify the cDNA insert. Restriction digests were performed with appropriate enzymes to both $(i)$ cut out the insert to check for overall expected size and (ii) cut specific sequences within the insert to verify both insert orientation and sequence. Appropriate clones were then grown in larger cultures, and phagemid DNA was purified from maxipreps using standard procedures and commercially available reagents (Plasmid Maxi Kit; Qiagen, Chatsworth, CA, USA). The cloned DNA was linearized with appropriate restriction enzymes (again verified by agarose gel electrophoresis) and extracted with an equal volume of phenol/chloroform/isoamyl alcohol (24: 25:1). The aqueous phase was incubated at room temperature for $2 \mathrm{~min}$ after the addition of $0.5 \mathrm{vol}$ of $7.5 \mathrm{M}$ ammonium acetate, $2 \mu \mathrm{L}$ of Pellet Paint ${ }^{\circledR}$ Co-Precipitant (Novagen, Madison, WI, USA) and 1 vol of $100 \%$ isopropanol. After centrifugation at $4^{\circ} \mathrm{C}$ for $15 \mathrm{~min}$ (ca. $12000 \times g$ ), the DNA pellet was washed with $500 \mu \mathrm{L}$ of $70 \%$ ethanol, air-dried and resuspended in TE buffer $(10 \mathrm{mM}$ Tris-HCl, 1 mM EDTA, pH 7.4). Purity and concentration of phagemid DNA was determined by UV spectroscopy in TE buffer. Phagemid DNA was then diluted to $10 \mu \mathrm{g} / \mathrm{mL}$ in $6 \times$ standard saline citrate (SSC) and stored at $-20^{\circ} \mathrm{C}$.

To make the membrane blots for the reverse Northern blots, the linearized DNA was removed from the freezer, extensively heat-denatured at $85^{\circ} \mathrm{C}$ for 10-15 min and slot-blotted by capillary pressure onto a nylon membrane using the manufacturer's specifications $(1 \mu \mathrm{g}$ per slot, Hybond ${ }^{\circledR}-\mathrm{N}$; Amersham Pharmacia Biotech, Piscataway, NJ, USA). The membrane was removed, UVcrosslinked and allowed to dry under dust-free conditions. The dried blots were stored under dry, clean conditions until used (typically within a few days to weeks).

\section{RNA Isolation and Purification}

From $\lambda$ phagemids: Both $\lambda 1.2$-pBS and $\lambda 2.5-\mathrm{pBS}$ phagemids were used as templates for separate $20-\mu \mathrm{L} \mathrm{T7}$ in vitro transcription reactions according to the manufacturer's instructions (AmpliScribe $^{\mathrm{TM}}$; Epicentre Technologies, Madison, WI, USA). After adding 4 vol of TE buffer made with diethyl pyrocarbonate (DEPC)-treated water, the RNA was extracted and precipitated as above. Full-length transcripts with 3' poly(A) tails were purified using oligo(dT) Dynabeads ${ }^{\circledR}$ (Dynal, Lake Success, NY, USA) according to the manufacturer's instructions. The purified poly(A) RNA was again extracted, precipitated and redissolved in TE buffer. UV spectrophotometry was used to determine RNA purity and quantity. Denaturing RNA gel electrophoresis was used to verify RNA size.

To determine copy number, the molecular weight of each full-length single transcript was first determined by multiplying the number of bases by 340 $\mathrm{Da} / \mathrm{base}$. The number of grams per single transcript was then determined by dividing the molecular weight by Avogadro's number. Concentrated RNA stocks were stored at $-80^{\circ} \mathrm{C}$ until use (within several months). Serial dilutions of concentrated stocks were used to obtain appropriate copy numbers of each poly(A) RNA.

\section{aRNA Amplification}

The overall scheme of the procedures outlined below (Figure 1) is similar to that described by Eberwine and colleagues (8), but some reagents and protocols have been modified. Whenever possible, master-mixtures were used. For the sensitivity studies, equal copies 
of the 1.2- and $2.5-\mathrm{kb}$ poly(A) $\lambda \mathrm{RNA}$ standards were used. Parallel samples were used as tracer reactions with a radiolabeled nucleotide to monitor various steps of the protocol (e.g., first- and second-strand cDNA syntheses and in vitro transcription). For the $3^{\prime}$ bias and cross-hybridization studies, only $2.5-\mathrm{kb}$ poly(A) $\lambda$ RNA was added to the reactions containing rat nerve RNA.

First round. First- and secondstrand syntheses were carried out using standard conditions as specified by the manufacturer using SUPERSCRIPT ${ }^{\mathrm{TM}} \mathrm{Re}$ verse Transcriptase (Life Technologies, Gaithersburg, MD, USA). Poly(A) RNA was primed with $100 \mathrm{ng}$ of a modified oligo(dT) primer [T7-oligo(dT)] containing the promoter sequence for T7 RNA polymerase (2). Firststrand cDNA was synthesized for $2 \mathrm{~h}$ at $42^{\circ} \mathrm{C}$. Second-strand synthesis was carried out using the Gubler-Hoffman reaction (4) for $2-4 \mathrm{~h}$ at $16^{\circ} \mathrm{C}$. The tubes were then placed on ice, and the double-stranded cDNA was extracted and precipitated as described above. After washing with $70 \%$ ethanol, the DNA was resuspended in $20 \mu \mathrm{L}$ DEPC-treated $\mathrm{H}_{2} \mathrm{O}$ and blunt-ended in a final volume of $25 \mu \mathrm{L}$ containing $20 \mathrm{mM}$ Trisbase, $\mathrm{pH} 7.5,10 \mathrm{mM} \mathrm{MgCl}_{2}, 5 \mathrm{mM}$ $\mathrm{NaCl}, 5 \mathrm{mM}$ dithiothreitol (DTT), 500 $\mu \mathrm{M}$ dNTPs and $1 \mathrm{U}$ E. coli DNA Polymerase I, large fragment (Klenow; Life Technologies) for $15 \mathrm{~min}$ at $37^{\circ} \mathrm{C}$.

In preparation for first-round in vitro transcription, the DNA was again extracted, precipitated twice (without further addition of Pellet Paint) and the pellet resuspended in $9 \mu \mathrm{L}$ as above. Double-precipitation was utilized to remove dNTPs that can interfere with in vitro transcription. The template was transcribed in $20-\mu \mathrm{L}$ reactions containing amplification buffer and $7.5 \mathrm{mM}$ NTPs (AmpliScribe), 10 mM DTT, 10 U RNasin ${ }^{\circledR}$ Ribonuclease Inhibitor (Promega) and 1000 U T7 RNA Polymerase (Epicentre Technologies) at $37^{\circ} \mathrm{C}$ for $8-12 \mathrm{~h}$. The poly(U) aRNA was extracted and precipitated twice to remove proteins and NTPs that can interfere with second-round, first-strand synthesis. Amplification was determined by dividing the total amount of aRNA synthesized by the amount of double-stranded coding sequence.

Second round. The purified poly-
(U) RNA was redissolved in $7.5 \mu \mathrm{L}$ of TE buffer and combined with $50 \mathrm{ng}$ of random hexamer primers, heated at $70^{\circ} \mathrm{C}$ for $10 \mathrm{~min}$, cooled on ice, and first-strand cDNA synthesis carried out as described above. The first-strand cDNA reaction was extracted, precipitated and resuspended as described above in $11.5 \mu \mathrm{L}$ of TE buffer. The RNA/DNA hybrid was then heated to $95^{\circ} \mathrm{C}$ for $3 \mathrm{~min}$ and placed on ice. Second-strand synthesis was performed in a total of $20 \mu \mathrm{L}$ containing $100 \mathrm{ng}$ T7oligo(dT), $20 \mathrm{mM}$ Tris-base, $\mathrm{pH}$ 7.5, 10 $\mathrm{mM} \mathrm{MgCl} 2,5 \mathrm{mM} \mathrm{NaCl}, 5 \mathrm{mM}$ DTT, $250 \mu \mathrm{M}$ dNTPs and $1 \mathrm{U}$ each of T4 DNA Polymerase and E. coli DNA Polymerase I, large fragment (Klenow) for $8-12 \mathrm{~h}$ at $16^{\circ} \mathrm{C}$. The cDNA (now with a functional $\mathrm{T} 7$ promoter site) was extracted, precipitated (without the further addition of Pellet Paint), resuspended in $20 \mu \mathrm{L}$ TE buffer and bluntended as described above.

In preparation for the second-round in vitro transcription, the DNA was again extracted, precipitated and the pellet resuspended in $10 \mu \mathrm{L}$ as above. The template was transcribed in $20-\mu \mathrm{L}$ reactions containing T7 TranscriptionPak $^{\mathrm{TM}} 1 \times$ Amplification Buffer (Epicentre Technologies), $500 \mu \mathrm{M} 3$-NTPs (ATP, GTP and UTP), $12.5 \mu \mathrm{M}$ CTP, $2.5 \mu \mathrm{M}[\alpha-32 \mathrm{P}] \mathrm{CTP}, 10 \mathrm{mM}$ DTT, $10 \mathrm{U}$ RNasin and $1000 \mathrm{U}$ T7 RNA polymerase at $37^{\circ} \mathrm{C}$ for $8-12 \mathrm{~h}$. The radiolabeled aRNA was extracted and precipitated twice, heat-denatured at $75^{\circ} \mathrm{C}$ for $5 \mathrm{~min}$ and added to the prehybridized reverse Northern blots.

\section{Hybridization, Washing Conditions and Analysis}

Blots were prehybridized at $42^{\circ} \mathrm{C}$ overnight in $5-10 \mathrm{~mL}$ of prehybridization solution containing $50 \% \mathrm{wt} / \mathrm{vol}$ formamide, $5 \times \mathrm{SSC}, 0.5 \% \mathrm{wt} / \mathrm{vol}$ sodium dodecyl sulfate (SDS), $1 \mathrm{mM} \mathrm{Na}$ pyrophosphate, $5 \times$ Denhardt's reagent and $100 \mu \mathrm{g} / \mathrm{mL}$ sheared salmon sperm DNA. Heat-denatured probe was added directly to the prehybridization solution and incubated at $42^{\circ} \mathrm{C}$ for $48 \mathrm{~h}$. The blots were removed and washed in large volumes as follows: twice in $2 \times$ SSC, $0.1 \%$ SDS for 15 min each at $42^{\circ} \mathrm{C}$; once in $0.2 \times \mathrm{SSC}, 1 \% \mathrm{SDS}$ for $15 \mathrm{~min}$ at $42^{\circ} \mathrm{C}$; and once in $0.1 \times \mathrm{SSC}$,
$0.1 \% \mathrm{SDS}$ for $30 \mathrm{~min}$ at $48^{\circ} \mathrm{C}$. Following washing, excess fluid was removed from the blots, and they were immediately wrapped in cellophane without drying. The blots were then exposed to a PhosphorImager ${ }^{\circledR}$ plate (Molecular Dynamics, Sunnyvale, CA, USA) for 24-48 h. The exposed plate was scanned by a PhosphorImager (Molecular Dynamics), and the amount of radioactivity was quantified using

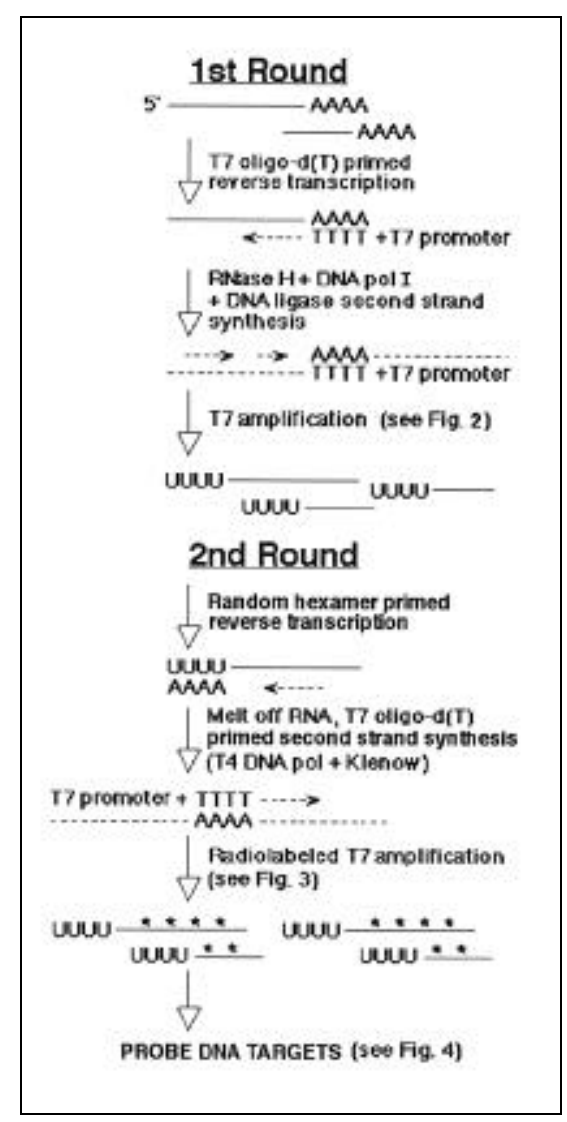

Figure 1. Overview of amplification protocol. RNA and cDNA are represented by solid and dashed lines, respectively. Vertical arrows indicate sequential enzymatic steps, and horizontal arrowheads indicate the direction of synthesis within each reaction. A primer consisting of the T7 RNA polymerase promoter sequence and oligo(dT) is used in the first round to reversetranscribe poly(A) RNA. Gubler-Hoffman second-strand synthesis creates cDNA with a functional T7 promoter. This template is then used for a T7-based in vitro transcription reaction (T7 amplification), resulting in up to 3000-fold amplification. RT in the second round is primed with random hexamers, and second-strand cDNA synthesis uses the T7-oligo(dT) primer, but uses different enzymes than in the first round. The resulting cDNA (with functional T7 promoter) is used for a radiolabeled in vitro transcription reaction, and the products are used to probe specific DNA targets on the reverse Northern blot. 
ImageQuant ${ }^{\mathrm{TM}}$ software (Molecular Dynamics). Only DNA targets that displayed signals that were at least two times the vector-only background were considered for further data analysis.

\section{RESULTS}

\section{Amplification Efficiency}

Nanogram quantities of the $\lambda 2.5$ and $\lambda 1.2-\mathrm{pBS}$ vectors were used to carry out a time-course analysis of the amplification efficiency under "cold" (first round) and "hot" (second round) conditions. Near full-length products were seen to increase up to 8-12 h (Figure 2A). Although full-length products were still quite abundant at $24 \mathrm{~h}$, there was increasing evidence of potential RNA degradation and/or synthesis of shorter products. Four nanograms of

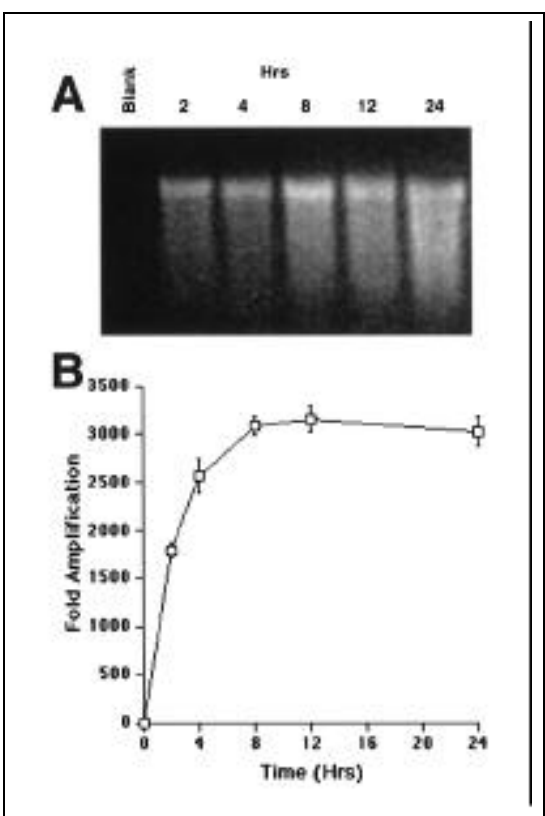

Figure 2. First-round T7 amplification. Four nanograms of cDNA template $(\lambda 2.5$-pBS) were used in a time-course study ( $2-24 \mathrm{~h}$ ) to model the first-round "cold" T7 in vitro transcription reaction conditions. Independent reactions were used for each time point. Following extraction and precipitation, proportional aliquots of the final reaction products were analyzed by both denaturing gel-electrophoresis (A) and UV spectrophotometry (B). The "blank" lane in Panel A represents a no-template reaction processed in parallel. Fold amplification was determined by dividing the total amount of aRNA synthesized by the amount of double-stranded coding sequence in $4 \mathrm{ng} \lambda 2.5$ pBS. Error bars $=$ standard error of the mean (SEM), $n=2$ reactions/time point. $\lambda 2.5$-pBS yielded approximately 3000 fold amplification during the firstround transcription reaction as quantified by spectrophotometric analysis (Figure 2B).

During the second-round amplification, the aRNA was radiolabeled. These reaction conditions necessarily involve limited amounts of the NTP used for labeling purposes. This results in production of less than full-length transcription products. Despite this limitation, scintillation counts of incorporated transcription products indicated approximately 1000 -fold amplification (data not shown). Electrophoretic analysis of radiolabeled reaction products showed an increase between 8 and $24 \mathrm{~h}$ when starting with picogram quantities of template (Figure 3A).

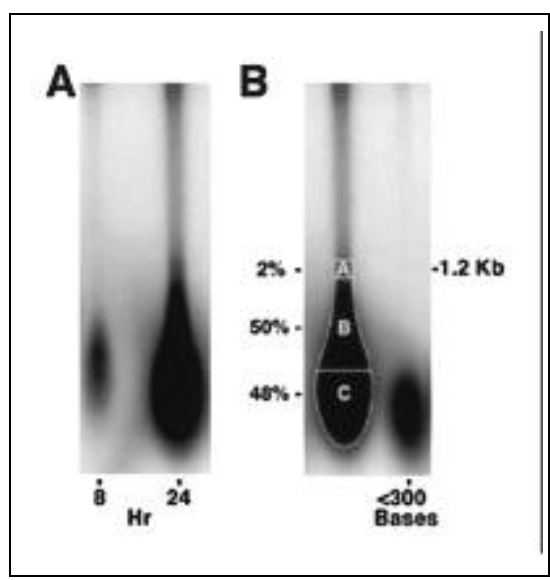

Figure 3. Second-round T7 amplification. Sixty picograms of a cDNA template $(\lambda 1.2-\mathrm{pBS})$ were used in a time-course study ( 8 and $24 \mathrm{~h}$ ) to model the second-round "hot" T7 in vitro transcription reaction conditions. Independent reactions were used for each time point, and equal proportions of each reaction were analyzed by denaturing gel electrophoresis. (A) Time course. There was a dramatic increase in the autoradiographic intensity of synthesized products from 8-24 h. Most of the products were less than fulllength at both time points (see size marker in Panel B), which is indicative of potential RNA degradation or the synthesis of aborted transcripts. (B) Transcript length analysis. To estimate the mobility of 300-base transcripts, a second aliquot of the aRNA from the 24-h time point was size-fractionated to purify RNA up to 300 bases (right-hand lane). The left-hand lane is the same image as shown in Panel A for the 24-h time point. Borders were drawn around the estimated full-length products (area A), the less than 300-base products (area C) and the region in between (area B). The relative signal intensity of these three areas was then determined using ImageQuant software, and the percentage of signal represented by each area is indicated.
Such analysis also showed that the majority of reaction products under these conditions were less than full-length (Figure 3B).

\section{Sensitivity of Detection and Amplifi- cation of Different Length mRNAs}

Equal copy numbers of both 1.2- and 2.5-kb $\lambda$ RNA internal standards were used in the same tubes as starting material for the aRNA procedure. The radiolabeled aRNA probe was hybridized to reverse Northern blots containing the $\lambda 1.2-$ and $\lambda 2.5-\mathrm{kbp}$ DNA targets and a target containing $400 \mathrm{bp}$ of nonoverlapping $\lambda D N A$ as an additional control for the possible nonspecific binding of radiolabeled probe to any $\lambda$ DNA target. As low as 10 copies of each $\lambda$ RNA could be reliably detected on the reverse Northern blots (Figure 4A). Signal intensities were equivalent for both the 2.5- and 1.2-kbp DNA targets, indicating similar amplification efficiencies for both poly(A) $\lambda$ RNAs (Figure 4B). This result is due to the normalization

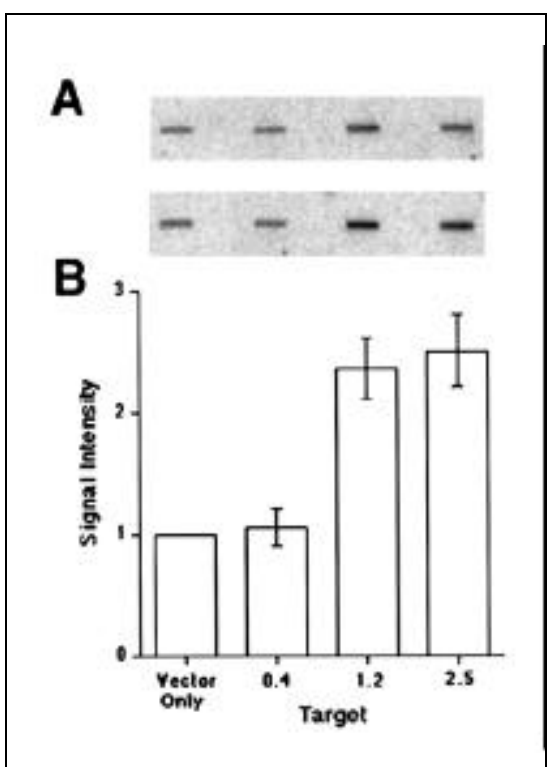

Figure 4. Sensitivity of detection. Ten copies each of the $\lambda 1$.2- and $\lambda 2.5-\mathrm{kb}$ RNAs were used as the starting material for 2 rounds of T7 amplification. (A) Images of reverse Northern blots from 2 independent experiments are shown. The 4 slots represent binding to the 4 DNA targets indicated on the X-axis of Panel B. (B) ImageQuant software was used to determine signal intensity of the 4 slots and was normalized to vector-only (pBS). There was no increase over vector-only signal for a nonoverlapping $0.4-\lambda$ DNA target. $n=2$, error bars $=$ SEM. 
process that occurs during the random hexamer priming of the second round of amplification (see Discussion).

\section{$3^{\prime}$ Bias of the Amplified Products}

There is an inherent bias to preferentially amplify the $3^{\prime}$ end of mRNAs during the aRNA procedure due to priming the first RT step with an oligo(dT)based primer. To investigate the extent of this potential problem, the $5^{\prime}$-most, 600 -bp fragment of $\lambda 2.5$-pBS was subcloned and used as a reverse Northern blot target along with full-length $\lambda 2.5$ pBS. To model typical experimental conditions, we used RNA isolated from single rat nerve samples. Animals were deeply anesthetized, and $1-\mathrm{cm}$ segments of sciatic nerve were removed and placed into FastPrep ${ }^{\mathrm{TM}}$ tubes (Bio 101, Vista, CA, USA). Following the manufacturer's protocol, the tissue was homogenized with RNA SAFE KITTM reagents (Bio 101) in the FastPrep apparatus, and total RNA was isolated, precipitated and subjected to DNase treatment to remove contaminating DNA. The RNA was spiked with poly(A) $2.5-\mathrm{kb} \lambda \mathrm{RNA}$ during the initial RT step and processed through 2 rounds of amplification as described above. Radioactive probe was applied to reverse Northern blots that contained both full-length and $5^{\prime} \lambda 2.5$-pBS targets. As expected, there was a diminution of the signal intensity for the $5^{\prime}$ target compared to full-length (Figure 5). However, the $5^{\prime}$ signal was approximately $50 \%$ of the full-length signal, suggesting that at least within a factor of two, this is not a severe problem for the procedure with poly(A) RNAs of at least $2.5 \mathrm{~kb}$.

\section{Potential Cross-Hybridization}

One of the major concerns with any slot/blot approach to study mRNA abundance is the issue of cross-hybridization among homologous mRNAs. To assess this problem for these internal standards, two identical reverse Northern blots were prepared. One of the blots was hybridized overnight during the prehybridization step with $10 \mu \mathrm{g}$ of cold antisense $2.5-\mathrm{kb} \lambda \mathrm{RNA}$. The next day, both blots were washed extensively with the prehybridization mixture to remove unbound $\lambda$ RNA. Radiolabeled probe was made from the same nerve template spiked with poly(A) $2.5-\mathrm{kb}$ $\lambda$ RNA as described above and was applied to both blots. The blot that had received the cold competitor during the prehybridization step displayed a near complete elimination of the $2.5-\mathrm{kbp}$ $\lambda$ DNA target signal (Figure 6), while the signal intensities from all other DNA targets on the blots were not affected (data not shown).

\section{DISCUSSION}

The results of the experiments reported in this manuscript show the utility of applying poly(A) $\lambda$ RNA internal standards to studies of mammalian gene expression. Such standards are easily produced and provide a powerful means for monitoring the amplification and detection sensitivity of the amplified aRNA procedure. In addition, the inclusion of such standards allows straightforward optimization of hybridization and blot washing conditions (i.e., stringency) as well as troubleshooting the various steps of the procedure.

To analyze conditions of first-round transcription reactions, we used the lowest amount of template that gave a reliable signal with ethidium bromide

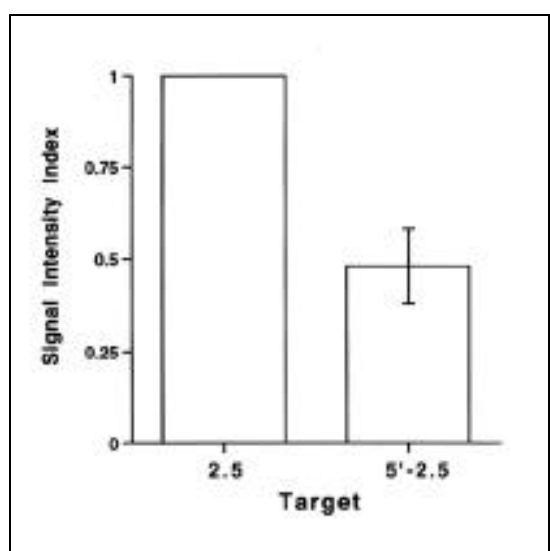

Figure 5. 3' Bias of amplified products. Radiolabeled probe from mRNA of single rat nerves spiked with $10^{5}$ copies of the poly(A) $\lambda 2.5-\mathrm{kb}$ RNA was hybridized to reverse Northern blots containing both the full-length $2.5 \lambda \mathrm{DNA}$ target (2.5) and a target containing the $5^{\prime}$-end fragment $\left(5^{\prime}-2.5\right)$. Signal intensity of the full-length 2.5 $\lambda$ DNA target was arbitrarily set to 1 , and the 5 end fragment was then expressed as a percentage of the signal of the full-length target. Results are from 4 independent experiments using 4 different nerve samples. Error bars $=$ SEM. staining (4 ng). The finding of thousandfold amplification efficiencies is substantially higher than that reported for more standard in vitro transcription reactions that use microgram quantities of DNA templates. We have found that even under the optimized conditions reported here, microgram quantities of DNA template yields approximately 450-fold amplification, while nanogram quantities (i.e., the range of mRNA expected from small tissue samples) yield approximately 3000 -fold amplification. Although we have not undertaken an exhaustive study of the linearity of amplification of the $\lambda$ RNA standards spiked into the initial RT step, we have varied the amount of standard added to single rat nerve templates by a hundredfold (105-10 7 copies) and have found a

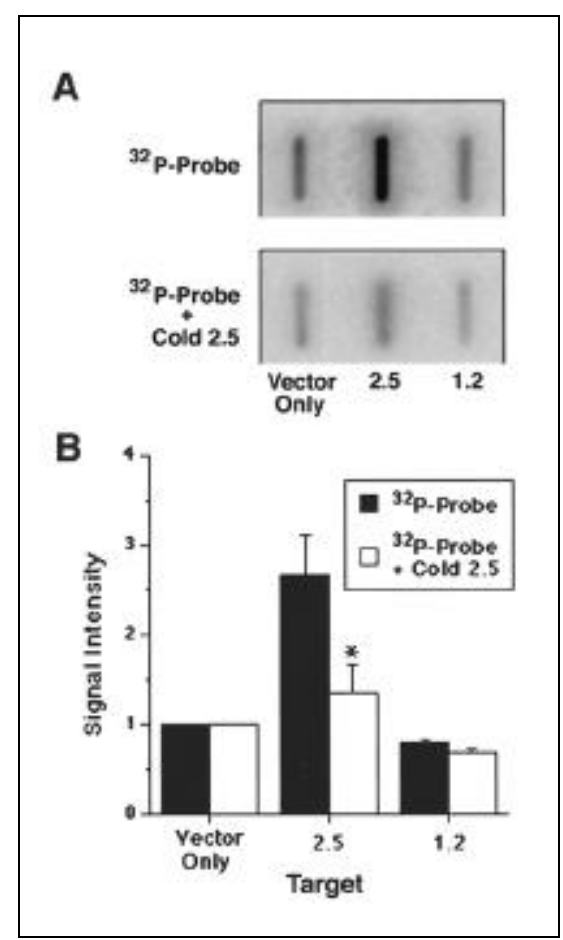

Figure 6. Cross-hybridization analysis. Radiolabeled probe was made from second-round cDNA nerve templates as described in Figure 5, which were spiked only with poly(A) $2.5-\mathrm{kb}$ $\lambda$ RNA. (A) Note the lack of cross-hybridization of the probe with the nonspecific 1.2 $\lambda$ DNA target (top panel). Also note the lack of signal for the $2.5 \lambda$ DNA target on the blot that was prehybridized overnight with cold antisense $2.5 \mathrm{~kb}$ $\lambda$ RNA (bottom panel). (B) The difference in signal intensity of the $2.5 \lambda$ DNA target due to prehybridization with cold antisense $2.5-\mathrm{kb} \lambda$ RNA was statistically significant; ${ }^{*}=P<0.05$ for two-tailed Student's $t$-test. Results are from 2 independent experiments using 2 different nerve samples. Error bars $=\mathrm{SEM}$. 
linear response of signal intensity on the final reverse Northern blots (data not shown). The addition of the $\lambda$ RNA standards did not influence the relative signal intensities of the other cDNA targets on the blot, and the amount of radioactive probe generated from nerve templates also followed the time course above (data not shown).

The amplification process during the radiolabeling reaction results in less than full-length transcription products due to limiting concentrations of one of the NTPs (Figure 3). To model these reaction conditions, we used $60 \mathrm{pg}$ of template, a number that is in the range of aRNA expected from a limited tissue sample following first-round amplification. Only a few percent of the transcription products were determined to be full-length by gel electrophoresis, yet the signal intensity for the target containing just the $5^{\prime}$-most, 600-bp fragment of the full-length $\lambda 2.5-\mathrm{kbp}$ target was only decreased by approximately $50 \%$ (Figure 5). This is explained by the increased radiolabel incorporated into full-length, or near full-length, transcripts compared with much shorter transcripts. For instance, if one simply looks at the extreme cases, a full-length transcript of $2.5 \mathrm{~kb}$ would have $8.3 \times$ the amount of radiolabel compared with a 300-base transcript. Thus the same signal intensity on the blot would be generated by $6 \%$ of full-length transcripts as compared with $50 \%$ of the transcripts being 300 bases. Such signal intensity differences could also take place on the blot if one cDNA target is many times larger than another. The possibility for such differential intensity of different size transcripts must be recognized when comparing the signal intensities of cDNA targets on the blot. Partial RNA degradation of fulllength transcripts would also increase the proportional signal intensity of shorter products on the RNA gel while still containing probe that would bind to the $5^{\prime}$ DNA target on the blot.

The $3^{\prime}$ bias that results from priming the initial RT step with a modified oligo(dT) is theoretically exacerbated by priming the cold aRNA products from the first round of amplification with random hexamers before the secondround $\mathrm{T} 7$ amplification (see below). Experimentally however, a cDNA target containing just the $5^{\prime}$-most, 600-bp fragment of the full-length, $\lambda 2.5-\mathrm{kbp}$ DNA displayed approximately half the intensity of the full-length target (Figure 5). This implies that, at least for our model templates, the aRNA procedure will amplify mRNA at least $2-2.5 \mathrm{~kb}$ in length without a significant $3^{\prime}$ bias. This result suggests that the relative autoradiographic intensity on the blot is reflective of the original abundance of the corresponding mRNA, despite whatever vagaries might exist because of the multiple priming and synthetic steps in the aRNA procedure, at least for these 2 model templates. The issue of $3^{\prime}$ bias must also be considered if the DNA targets on the blot differ substantially in terms of which portion of the mRNA they represent (i.e., $3^{\prime}$ or $5^{\prime}$ ). 
A similar problem can be imagined in terms of the amplification of two mRNA species that differed in size by a factor of two; one might expect the longer mRNA to give a more intense signal on the final reverse Northern blot. This is not the case due to the normalization process that takes place due to priming the RT step of the second round of amplification with random hexamers. If both mRNA species resulted in forming full-length templates following the initial first- and secondstrand cDNA syntheses, the first-round aRNA transcription products could still have a size difference of a factor of two. However, during the priming of these aRNA transcription products with random hexamers for second-round RT, each transcript would contain on average a priming site every $1 \mathrm{~kb}$. Reverse transcription is not a displacement synthesis, and therefore, only the $5^{\prime}$-most priming site on the aRNA template [representing the $3^{\prime}$-most area of the original poly(A) RNA] will be able to copy the $5^{\prime}$ sequence of the aRNA template, and thus, only this priming event will result in a cDNA that now contains a $3^{\prime}$ poly(A) tail. During the subsequent second-strand synthesis, only those cDNAs with a $3^{\prime}$ poly(A) tail will be primed with the modified oligo(dT) primer and result in fully competent templates for the second in vitro transcription reaction. The result is that similar copy numbers of even widely different size mRNA species give comparable intensities on the final reverse Northern blot (Figure 4). Further evidence that such normalization of length takes place is suggested by the similarity of gels analyzing the radioactive probe from second-round amplifications. Such gels show roughly similar length products (similar to Figure 3B) even when starting with nerve mRNAs ranging from a few hundred to several kilobases in size (data not shown).

The issue of cross-hybridization was approached by analyzing a cold competition of the 2.5-kb $\lambda$ RNA internal standard (Figure 6). Experiments such as this show that the $2.5-\mathrm{kb} \lambda \mathrm{RNA}$ is not cross-hybridizing to any of the other cDNA targets on the reverse Northern blot. Such control experiments are especially pertinent when the cold competitor is known to have a high degree of sequence homology with one or more cDNA targets represented on the reverse Northern blot.

In conclusion, our results show the utility of using poly(A) $\lambda$ RNA internal standards to monitor the sensitivity and efficiency of the amplified aRNA procedure to generate expression profiles of relative mRNA levels from limited amounts of starting material. Under the optimized conditions reported in this paper, detection levels down to 10 copies of an RNA standard are possible. The potential problems of the procedure include unequal amplification of mRNA populations, $3^{\prime}$ bias due to oligo(dT) priming and cross-hybridization of homologous mRNAs on the final reverse Northern blot. The use of $\lambda$ RNA internal standards has shown that none of these potential problems are serious impediments to using this semiquantitative procedure to simultaneously measure the relative levels of multiple mRNAs. These standards can also help to verify the validity of traditional "housekeeping" genes that may or may not change in different tissue types under different conditions.

\section{ACKNOWLEDGMENTS}

This work was supported by the US VA Medical Research Service (R.D.M.), NIH-NS22404-12 (R.D.M.), Brain Injury Research Fund (G.A.R.), NIH-P01 NS14899 (G.A.R.) and NS16433 (G.A.R.). We thank Dr. John Hemperly for a critical reading of the manuscript and Dr. Jim Eberwine for many helpful discussions.

\section{REFERENCES}

1.Crino, P. and J.H. Eberwine. 1996. Molecular characterization of the dendritic growth cone: regulated mRNA transport and local protein synthesis. Neuron 17:1173-1187.

2.Eberwine, J. 1996. Amplification of mRNA populations using aRNA generated from immobilized oligo(dT)-T7 primed cDNA. BioTechniques 20:584-591.

3.Eberwine, J., P. Crino and M. Dichter. 1995. Single-cell mRNA amplification: implications for basic and clinical neuroscience. The Neuroscientist 1:200-211.

4.Gubler, U. and B.J. Hoffman. 1983. A simple and very efficient method for generating cDNA libraries. Gene 25:263-269.

5.Mackler, S.A. and J.H. Eberwine. 1993. Diversity of glutamate receptor subunit mRNA expression with live hippocampal CA1 neurons. Mol. Pharmacol. 44:308-315.

6.Nair, S.M. and J.H. Eberwine. 1994. Molecular correlates of corticosterone action in hippocampal subregions. Methods Neurosci. 22:314-329.

7.Robinson, G.A. and R.D. Madison. 1997. Sensitivity and amplification of gene expression profiling. Soc. Neurosci. Abst. 23:1722.

8.Van Gelder, R.N., M.E. Zastrow, A. Yool, W.C. Dement, J.D. Barchas and J.H. Eberwine. 1990. Amplified RNA synthesized from limited quantities of heterogenous cDNA. Proc. Natl. Acad. Sci. USA 87:1663-1667.

9. Wodicka, L., H. Dong, M. Mittmann, M.-H. Ho and D.J. Lockhart. 1997. Genome-wide expression monitoring in Saccharomyces cerevisiae. Nature Biotechnol. 15:1359-1367.

Received 14 January 1998; accepted 30 March 1998.

Address correspondence to:

Dr. Roger D. Madison

Division of Neurosurgery

P.O. Box 2609

Medical Sciences Research Building

Duke University Medical Center

Durham, NC 27710, USA

Internet: madis001@mc.duke.edu 